\title{
Morphometric skin characteristics dependent on chronological and biological age: the Leiden Longevity Study
}

\author{
Mariette E. C. Waaijer • David A. Gunn • Sharon D. Catt • Michael van Ginkel • \\ Anton J. M. de Craen • Nicole M. Hudson • Diana van Heemst • \\ P. Eline Slagboom • Rudi G. J. Westendorp • Andrea B. Maier
}

Received: 16 June 2011 / Accepted: 25 August 2011 / Published online: 10 September 2011

(C) The Author(s) 2011. This article is published with open access at Springerlink.com

\begin{abstract}
The effect of chronological age on skin characteristics is readily visible, and its underlying histological changes have been a field of study for several years. However, the effect of biological age (i.e. a person's rate of ageing compared to their chronological age) on the skin has so far only been studied in facial photographs. Skin biopsies obtained from middle-aged offspring of nonagenarian siblings that are genetically enriched for longevity were compared to their partners
\end{abstract}

Electronic supplementary material The online version of this article (doi:10.1007/s11357-011-9314-5) contains

supplementary material, which is available to authorized users.

M. E. C. Waaijer · A. J. M. de Craen - D. van Heemst •

R. G. J. Westendorp · A. B. Maier $(\bowtie)$

Department of Gerontology and Geriatrics,

Leiden University Medical Center,

2300 RC Leiden, The Netherlands

e-mail: a.b.maier@lumc.nl

D. A. Gunn · S. D. Catt $\cdot$ M. van Ginkel - N. M. Hudson Unilever,

Colworth Science Park,

Sharnbrook, Bedfordshire, UK

A. J. M. de Craen - D. van Heemst • R. G. J. Westendorp •

A. B. Maier

Netherlands Consortium for Healthy Aging,

Leiden University Medical Center,

2300 RC Leiden, The Netherlands

P. E. Slagboom

Department of Molecular Epidemiology,

Leiden University Medical Center,

2300 RC Leiden, The Netherlands who represent the general Dutch population. Though of the same chronological age, the offspring were previously observed to be of a younger biological age than their partners. The biopsies were analysed on several aspects epidermal and elastic fibre morphology. We investigated whether these skin characteristics were dependent on chronological age, familial longevity (the difference between the offspring and partners) and Framingham heart risk scores, adjusted for external stressors. A decreased thickness and flattening of the epidermis as well as an increased amount of elastic fibres in the reticular dermis were observed with chronological age $(P<0.001, P<0.001$ and $P=0.03$, respectively), but no effect of familial longevity was found. The Framingham heart risk score was associated with some skin characteristics. A slower rate of skin ageing does not mark offspring from nonagenarian siblings. Epidermal and elastic fibre morphometric characteristics are not a potential marker for familial longevity in middleaged subjects enriched for familial longevity.

Keywords Skin ageing Chronological age · Biological age $\cdot$ Familial longevity $\cdot$ Epidermis $\cdot$ Elastic fibres

\section{Introduction}

As the largest organ of the human body, the skin constitutes an important functional and cosmetic interface with the exterior world. The disintegration 
of this protective barrier with age is partly visible as wrinkling and loss of elasticity. Although ageing is associated with a general progressive loss of skin integrity, significant inter-individual differences exist. Apart from the cosmetic aspect, these inter-individual differences provide a potential biomarker for biological age as a lower perceived age based on facial photographs is associated with survival, even after correction for gender and chronological age (Christensen et al. 2009). The inter-individual differences in skin features can be partly explained by external factors such as smoking, alcohol and sun damage (Warren et al. 1991). However, there is increasing evidence that many of these differences in perceived age are genetically determined (Gunn et al. 2009; Christensen et al. 2009).

Wrinkling and the loss of elasticity are the result of several age-related changes in the underlying histological structure. The epidermis shows a flattening at the epidermal-dermal junction and a decreasing thickness with increasing age (Fenske and Lober 1986; Lavker et al. 1986; Fenske and Conard 1988; Kurban and Bhawan 1990). Within the dermis, the amount and length of mature elastic fibres in the papillary dermis have been found to increase with chronological age (Robert et al. 1988). In the reticular dermis, the length of elastic fibres and the percentage of elastin at the upper inner arm have all been found to increase with age (Robert et al. 1988; Frances et al. 1990). In addition, smoking has been positively correlated with an increased number and amount of elastic fibres in the reticular dermis of the upper inner arm (Just et al. 2007), highlighting the importance of controlling for confounding factors in studies of skin morphometric characteristics.

Within the present study, we have investigated the effect of chronological and biological age on epidermal histologic characteristics and morphology of elastic fibres in the upper inner arm. To compare with biological age, we used two established markers for biological age, the Framingham cardiovascular disease (CVD) risk score (D'Agostino et al. 2008) and familial longevity. Familial longevity was defined as differences between middle-aged offspring from longlived nonagenarian siblings and, as controls, their partners. Though of the same chronological age, these offspring have been previously observed to be of a younger biological age than their partners as reflected by their lower mortality rate, beneficial glucose and lipid metabolism, preservation of insulin sensitivity and resistance to cellular stress (Schoenmaker et al. 2006; Westendorp et al. 2009; Wijsman et al. 2010; Dekker et al. 2009). We questioned whether a slower rate of skin ageing marks middle-aged offspring of nonagenarian siblings compared to their partners.

\section{Materials and methods}

Study cohort

In the Leiden Longevity Study, genetically enriched subjects for familial longevity are compared with their partners to determine the genetic factors contributing to longevity. Four hundred and twenty-one families consisting of 943 long-lived Caucasian siblings with 1,671 of their offspring and 745 of the partners thereof were included in this cohort. The offspring carries on average $50 \%$ of the genetic propensity of their long-lived parent (Schoenmaker et al. 2006). Their partners, with whom most have shared the same environment for decades were included as matched controls. There was no selection on demographic or health characteristics (Schoenmaker et al. 2006; Westendorp et al. 2009). The current study includes 150 offspring and 150 partners thereof, from whom skin biopsies were taken in the period from November 2006 to May 2008. Of these 300 subjects, one subject eventually refused to have a biopsy taken, and the biopsies of 13 subjects were excluded after quality check. Therefore, for the epidermis and layer 1 of the dermis, data were available on 286 subjects. Nine subjects lacked data on layers two to five of the dermis and were therefore excluded from that analysis. The Medical Ethical Committee of the Leiden University Medical Center approved the study. Declaration of Helsinki protocols were followed, and written informed consent was obtained from all participants.

Morphometric analysis of the skin

Skin biopsies $(4 \mathrm{~mm})$ were taken from the sunprotected site of the inner upper arm and fixed in formalin (Sigma) overnight (18-24 h). After fixation, the biopsies were washed in fresh phosphate-buffered saline twice and then dehydrated in $70 \%$ alcohol and stored at room temperature. The samples were embedded in paraffin wax and cut into sections of 
$4 \mu \mathrm{m}$. Two sections of each biopsy were stained with Orcein dye. Haematoxylin and eosin staining (Sigma) was performed for quality checking purposes. A Leica DM 1000 microscope, with a Leica DFC420 Camera and Leica Application Suite Software was used to capture images of the slides with an objective magnification of $\times 10$. Image capture and analysis were performed blind to age, gender and the offspring/partner status of the subjects. To analyse the image data semi-automatically, image analysis software was constructed. The stratum corneum and epidermal-dermal boundary in the image were automatically delineated by the software. The epidermis was identified by its darker nature using an intensity threshold. The stratum corneum epidermis boundary was identified through absolute intensity and degree of striation (due to its layered nature) which was quantified using a variance filter. Thereafter, manual refinement was performed to ensure accurate delineation of the epidermal boundaries. In addition, manual exclusion of regions within the images were performed if they contained large structures (hair follicles, blood vessels, glands) or large areas of non-dermis i.e. blank areas, large gaps or tears in tissue and clusters of small non-dermis areas. Morphometric measurements of the epidermis and elastic fibres are depicted in Fig. 1. Epidermal measurements included the thickness of the epidermis derived from the area covered by epidermis divided by the length of the epidermal-dermal junction (EDJ). Furthermore, the curvature in the epidermis was defined as the ratio of a straight line between the horizontal margins of the epidermis over the length of the stratum corneumepidermis boundary (SEB). Within the dermis, the area covered by the elastic fibres, their number (both corrected for the size of image area they were measured in) and the morphometrical characteristics of individual elastic fibres were measured. These morphometrical characteristics included the area (micrometre squared), length (micrometre), thickness (micrometre) and the curl of an elastic fibre (defined by the ratio between its length and the calliper dimension). The elastic fibres were identified by applying an intensity threshold on the green channel of the red-green-blue colour image. Resolving the measurements by depth was dealt with by using the epidermal-dermal junction as the zero-depth reference point. The dermis was divided into depth layers of 100 micrometre. For each pixel on the epidermal- dermal junction, the corresponding pixel 100 micrometre lower on the $y$-axis was determined. Layer 1 represents the papillary dermis and layers 2 to 5 the reticular dermis. Nine biopsies had too little depth to supply data for layers 2 to 5 .

\section{Potential confounders}

For each subject, demographic characteristics and facial photographs as well as factors known to influence skin characteristics were available (e.g. smoking and alcohol use). To control for potential sun-exposure effects on the arm, the photographs of the face were assessed by a dermatologist blind to age, gender and offspring or partner status to determine photo/sun-damage scores (Griffiths et al. 1992; Gunn et al. 2009). This nine-point (no photodamage to severe photodamage) photonumeric scale assesses the severity of cutaneous photodamage. Information on medical history was obtained from the participants' treating physicians, including history of myocardial infarction, stroke, hypertension, diabetes, malignancy, rheumatoid arthritis and chronic obstructive pulmonary disease (COPD). Information on medication use was obtained from the participants' pharmacists and divided in categories composed of anti-thrombotic, anti-COPD, anti-diabetic, other endocrine and nervous system and cardiovascular drugs including lipid lowering medication. Total cholesterol and HDL-cholesterol used for the Framingham CVD risk score were measured in non-fasted serum samples using the Hitachi Modular P 800 from Roche, Almere, The Netherlands.

\section{Statistics}

All analyses were performed using SPSS 16.0 data editor software. The association of skin morphometric characteristics with chronological age and with offspring and partners status was analysed by linear regression using three different models. Chronological age: model 1 was adjusted for gender, model 2 was adjusted for the same covariates as for model 1 with further adjustment for external factors (smoking, alcohol and sun damage), model 3 additionally for the number of co-morbidities plus the number of medication categories. Familial longevity, comparing offspring (0) and partners (1): model 1 was adjusted for age and gender; models 2 and 3 were similar to those of chronological age. 


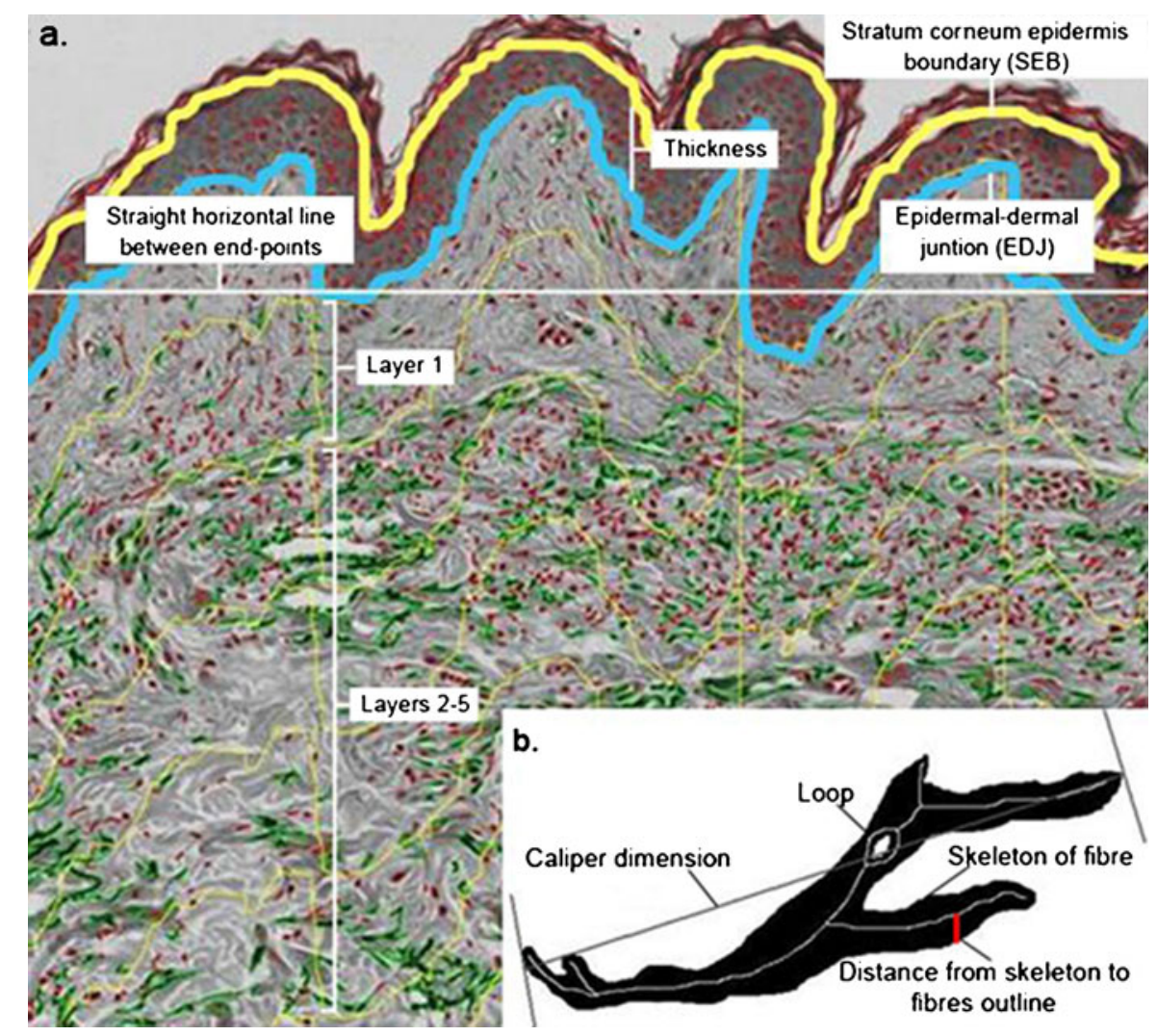

Fig. 1 Measurements on the epidermis and dermis. a Thickness based on the EDJ: area covered by epidermis (area between the yellow and light blue lines) divided by the EDJ (light blue) length given in micrometre. Curvature based on the stratum SEB: the ratio of the length of the straight horizontal line between the margins (white) and the length of the SEB (yellow). Area covered by elastic fibres (green: in-plane, red: end-on) given in micrometre squared. Layer 1: papillary dermis, layers 2 to 5 : reticular dermis. b Area of a single

To study the association between cardiovascular disease risk and morphometric characteristics of the skin, the Framingham CVD risk score was calculated as previously described (D'Agostino et al. 2008). Model 1 of the linear regression consisted of the 10 -year risk prediction of cardiovascular disease, model 2 adjusted for the offspring/partner status, model 3 was additionally adjusted for sun damage. $P$ values of less than 0.05 were considered to be significant.

\section{Results}

In total, 286 subjects were included in the analysis after a quality check of the biopsies, consisting of 141 elastic fibre: amount of area the fibre covers (micrometre squared). Length of an elastic fibre: length of the skeleton of the fibre (white) without half of the loop size if present plus for each end-point of the line the distance to the nearest point on the fibre's outline (micrometre squared). Thickness of an elastic fibre: ratio between size and length (nanometre). Curl of an elastic fibre: the ratio between the fibre's length and the maximum calliper dimension

males and 145 females aged between 44 and 81 years. The characteristics of the subjects are summarized in Table 1. The offspring had experienced less agerelated diseases such as myocardial infarction, hypertension and type 2 diabetes mellitus. Use of hormonal replacement therapy was not different between the offspring and partners; however, menopausal state differed significantly. Female partners were more frequently premenopausal compared to female offspring, most likely due to their younger age.

Table 2 shows the association between chronological age and morphometric characteristics of the epidermis and elastic fibres in the dermis with and without adjustment for potential confounders (estimates are given in the supplementary Table 1). With 
Table 1 Characteristics of subjects

Values are given as mean (SD) if not otherwise stated. Medication categories composed of anti-thrombotic, anti-COPD, anti-diabetic, other endocrine and nervous system and cardiovascular drugs (including lipid lowering medication). Photodamage was determined by scoring photographs of subjects with a nine-point photonumeric standard scale (no photodamage to severe photodamage)

$B M I$ body mass index, $M I$ myocardial infarction, $\mathrm{CVA}$ cerebrovascular accident, $C O P D$ chronic obstructive pulmonary disease, $S D$ standard deviation

${ }^{\text {a }} N=243$

${ }^{\mathrm{b}} N=182$

${ }^{\mathrm{c}} N=58$ and 80 (offspring, partners)

${ }^{\mathrm{d}} N=268$

${ }^{\mathrm{e}} N=263$

\begin{tabular}{|c|c|c|}
\hline & $\begin{array}{l}\text { Offspring } \\
N=143\end{array}$ & $\begin{array}{l}\text { Partners } \\
N=143\end{array}$ \\
\hline \multicolumn{3}{|l|}{ Demographic data } \\
\hline Female, \% & 43.4 & 58 \\
\hline Age, years & $63.3(6.0)$ & $63.2(7.1)$ \\
\hline \multicolumn{3}{|l|}{ Body composition } \\
\hline Height, cm & $172.2(9.1)$ & $170.7(8.6)$ \\
\hline BMI, $\mathrm{kg} / \mathrm{m}^{2}$ & $26.6(3.8)$ & $26.8(4.1)$ \\
\hline Fat, \% & $28.4(8.0)$ & $31.4(8.2)$ \\
\hline Lean mass, $\%$ & $67.5(7.6)$ & $64.6(7.7)$ \\
\hline \multicolumn{3}{|l|}{ Co-morbidities, no. $(\%)^{\mathrm{a}}$} \\
\hline MI & $1(0.8)$ & $4(3.1)$ \\
\hline CVA & $2(1.6)$ & $6(4.7)$ \\
\hline Hypertension & $28(22.0)$ & $38(29.7)$ \\
\hline Diabetes mellitus & $4(3.2)$ & $13(10.2)$ \\
\hline Malignancy & $4(3.1)$ & $7(5.6)$ \\
\hline COPD & $7(5.5)$ & $4(3.1)$ \\
\hline Rheumatoid arthritis & $0(0.0)$ & $0(0.0)$ \\
\hline Number of co-morbidities $(0-7)^{\mathrm{a}}$ & $0.3(0.6)$ & $0.6(0.8)$ \\
\hline Framingham heart risk ${ }^{\mathrm{b}}$ & $16.8(8.2)$ & $16.5(9.1)$ \\
\hline \multicolumn{3}{|l|}{ Medication } \\
\hline Medication categories $^{\mathrm{a}}$ & $0.6(0.8)$ & $0.8(1.0)$ \\
\hline Hormonal replacement therapy (former and current), no. $(\%)^{\mathrm{c}}$ & $8(14.0)$ & $9(11.4)$ \\
\hline \multicolumn{3}{|l|}{ Intoxications $^{\mathrm{d}}$} \\
\hline Users of alcohol, no. (\%) & $109(78.4)$ & $110(79.1)$ \\
\hline Former and/or current smoking, no. $(\%)$ & $85(61.6)$ & $100(71.9)$ \\
\hline Photodamage score ${ }^{\mathrm{e}}$ & $4.8(1.2)$ & $4.8(1.2)$ \\
\hline \multicolumn{3}{|l|}{ Menopausal status, no. $(\%)^{\mathrm{c}}$} \\
\hline Premenopausal & $0(0.0)$ & $7(8.8)$ \\
\hline Perimenopausal & $0(0.0)$ & $5(6.3)$ \\
\hline Postmenopausal & $58(100.0)$ & $68(85.0)$ \\
\hline
\end{tabular}

chronological age, the epidermal thickness significantly decreased whereas the curvature parameter increased significantly, indicating a flattening of the epidermis (both $P<0.001$ ). The decreasing thickness and the overall flattening of the epidermis with chronological age remained significant after adjustment for gender, external factors (smoking, alcohol and sun damage) and health status $(P=0.01$ and $P=0.001$, respectively). In the papillary dermis (layer 1$)$, a trend towards a larger area covered by elastic fibres $(P=0.06)$, a higher number of elastic fibres $(P=0.07)$, a larger area covered by a single elastic fibre $(P=0.05)$, an increased length of an elastic fibre $(P=0.08)$ and more curliness of an elastic fibre $(P=0.02)$ with chronolog- ical age was observed. However, these findings were diminished after adjustment for external factors and health status. The area covered by the elastic fibres and the number of elastic fibres in the reticular dermis (layers 2 to 5) were positively correlated with chronological age $(P=0.001$ and $P<0.001$, respectively). The increase in number of elastic fibres remained significant after adjustment for potential confounders $(P=0.03)$. None of the other elastic fibre morphometric characteristics were significantly associated with chronological age.

Table 3 shows the effect of familial longevity status, i.e. offspring and partner comparison, on the epidermal and elastic fibre morphometric character- 
Table 2 Chronological age and morphometric skin characteristics

\begin{tabular}{|c|c|c|c|c|c|c|c|}
\hline & \multicolumn{4}{|c|}{ Chronological age (years) } & \multirow{2}{*}{$\begin{array}{l}P \text { value } \\
\text { Model } 1\end{array}$} & \multirow{2}{*}{$\begin{array}{l}P \text { value } \\
\text { Model } 2\end{array}$} & \multirow{2}{*}{$\begin{array}{l}P \text { value } \\
\text { Model } 3\end{array}$} \\
\hline & $\begin{array}{l}\text { 1st quartile } \\
\mathrm{M}=45.5-59.9 \\
\mathrm{~F}=44.1-58.5\end{array}$ & $\begin{array}{l}\text { 2nd quartile } \\
M=59.9-64.6 \\
F=58.5-62.4\end{array}$ & $\begin{array}{l}\text { 3rd quartile } \\
\mathrm{M}=64.6-68.8 \\
\mathrm{~F}=62.4-66.8\end{array}$ & $\begin{array}{l}\text { 4th quartile } \\
\mathrm{M}=68.8-81.3 \\
\mathrm{~F}=66.8-78.3\end{array}$ & & & \\
\hline \multicolumn{8}{|l|}{ Epidermis $^{\mathrm{a}}$} \\
\hline Thickness based on EDJ, $\mu \mathrm{m}$ & $42.4(0.69)$ & $42.6(0.88)$ & $40.2(0.76)$ & $38.2(0.78)$ & $<0.001$ & $<0.01$ & 0.01 \\
\hline Curvature based on $\mathrm{SEB}^{\mathrm{b}}$ & $803.2(8.50)$ & $824.5(6.70)$ & $849.9(7.52)$ & $839.0(9.41)$ & $<0.001$ & 0.001 & 0.001 \\
\hline \multicolumn{8}{|l|}{ Dermis } \\
\hline \multicolumn{8}{|l|}{ Elastic fibres, layer $1^{\mathrm{a}}$} \\
\hline Area covered by fibres ${ }^{b}$ & $23.7(1.80)$ & $28.0(2.33)$ & $27.9(2.99)$ & $29.1(2.70)$ & 0.06 & 0.26 & 0.67 \\
\hline Number of fibres & $43.4(3.92)$ & $53.6(5.21)$ & $50.9(5.01)$ & $52.8(5.06)$ & 0.07 & 0.18 & 0.59 \\
\hline Area of a single fibre, $\mu \mathrm{m}^{2}$ & $27.5(0.79)$ & $28.7(0.96)$ & $29.1(1.04)$ & $29.9(1.42)$ & 0.05 & 0.35 & 0.68 \\
\hline Length of a fibre, $\mu \mathrm{m}$ & $15.3(0.41)$ & $15.8(0.43)$ & $16.2(0.44)$ & $16.0(0.50)$ & 0.08 & 0.30 & 0.66 \\
\hline Thickness of a fibre, $\mathrm{nm}$ & $1928(32.6)$ & $1886(21.4)$ & $1893(15.6)$ & $1910(23.0)$ & 0.43 & 0.31 & 0.42 \\
\hline Curl of a fibre ${ }^{b}$ & $926.1(4.97)$ & $906.1(8.10)$ & $907.1(5.73)$ & $907.3(7.06)$ & 0.02 & 0.10 & 0.24 \\
\hline \multicolumn{8}{|l|}{ Elastic fibres, layer $2-5^{\mathrm{c}}$} \\
\hline Area covered by fibres & $57.3(2.76)$ & $67.1(3.38)$ & $71.5(3.92)$ & $72.5(4.84)$ & 0.001 & 0.02 & 0.14 \\
\hline Number of fibres & $85.3(3.47)$ & $95.6(3.38)$ & $103.2(4.16)$ & $104.4(4.84)$ & $<0.001$ & 0.01 & 0.03 \\
\hline Area of a single fibre, $\mu \mathrm{m}^{2}$ & $47.8(1.50)$ & $51.4(2.77)$ & $51.3(1.73)$ & $49.2(2.61)$ & 0.29 & 0.31 & 0.82 \\
\hline Length of a fibre, $\mu \mathrm{m}$ & $20.9(0.47)$ & $21.5(0.68)$ & $21.8(0.49)$ & $20.6(0.76)$ & 0.68 & 0.70 & 0.74 \\
\hline Thickness of a fibre, $\mathrm{nm}$ & $2153(18.2)$ & $2177(22.3)$ & $2175(18.3)$ & $2155(25.8)$ & 0.35 & 0.26 & 0.74 \\
\hline Curl of a fibre ${ }^{b}$ & $909.4(4.02)$ & $903.5(4.73)$ & $902.5(5.45)$ & $898.3(8.40)$ & 0.49 & 0.97 & 0.98 \\
\hline
\end{tabular}

Values are given as mean (SE). $P$ values are derived from linear regression analysis. Model 1: gender adjusted, model 2: as model 1 plus external factors (smoking, alcohol and sun damage), model 3: as model 2 plus health status (no. of co-morbidities, no. of medication categories). Estimates of the different models are given in supplementary Table 1

$M$ male, $F$ female, EDJ epidermal-dermal junction, $S E B$ stratum corneum epidermis boundary

a 1 st quartile: $N=71,2$ nd quartile: $N=72,3$ rd quartile $N=72$, 4th quartile: $N=71$

$\mathrm{b} \times 10^{3}$

c 1st quartile: $N=69,2$ nd quartile: $N=72,3$ rd quartile $N=68,4$ th quartile: $N=68$

istics adjusted for potential confounders (estimates are given in the supplementary Table 2). No differences between offspring and partners were observed in any of the epidermal and elastic fibre characteristics before and after adjusting for chronological age, gender, external factors and health status. Stratification by chronological age did not change the results (data not shown).

To determine whether skin morphometric characteristics were associated with cardiovascular disease risk, epidermal and elastic fibre characteristics were related to the Framingham CVD risk scores (10-year risk predictions for cardiovascular disease, FHR). The results are shown in supplementary Tables 3 and 4 for the epidermis and elastic fibres, respectively. Epidermal and reticular elastic fibre (layers 2 to 5) morphometric characteristics were not significantly associated with the cardiovascular risk profile. The area covered by elastic fibres and the number of elastic fibres of the papillary dermis (layer 1) were negatively associated with the Framingham CVD risk scores (both $P=0.10$ ). Furthermore, the length of an elastic fibre and the area covered by an elastic fibre were significantly negatively related to the Framingham CVD risk scores in the papillary dermis after adjustment for potential confounders (layer 1 , both $P=0.01$ ).

\section{Discussion}

In the present study, we showed changes in morphometric characteristics of the epidermis: a decreased 
Table 3 Comparison of offspring and partners for morphometric skin characteristics

\begin{tabular}{|c|c|c|c|c|c|}
\hline & Offspring & Partners & $\begin{array}{l}P \text { value } \\
\text { Model } 1\end{array}$ & $\begin{array}{l}P \text { value } \\
\text { Model } 2\end{array}$ & $\begin{array}{l}P \text { value } \\
\text { Model } 3\end{array}$ \\
\hline \multicolumn{6}{|l|}{ Epidermis $^{\mathrm{a}}$} \\
\hline Thickness based on EDJ, $\mu \mathrm{m}$ & $40.9(0.56)$ & $40.9(0.58)$ & 0.94 & 0.46 & 0.87 \\
\hline Curvature based on $\mathrm{SEB}^{\mathrm{b}}$ & $832.2(5.44)$ & $826.2(6.28)$ & 0.62 & 0.22 & 0.86 \\
\hline \multicolumn{6}{|l|}{ Dermis } \\
\hline \multicolumn{6}{|l|}{ Elastic fibres, layer $1^{\mathrm{a}}$} \\
\hline Area covered by fibres ${ }^{b}$ & $27.1(1.89)$ & $27.2(1.64)$ & 0.70 & 0.35 & 0.56 \\
\hline Number of fibres & $49.9(3.55)$ & $50.5(3.29)$ & 0.91 & 0.91 & 0.91 \\
\hline Area of a single fibre, $\mu \mathrm{m}^{2}$ & $29.1(0.83)$ & $28.5(0.69)$ & 0.31 & 0.18 & 0.35 \\
\hline Length of a fibre, $\mu \mathrm{m}$ & $16.0(0.32)$ & $15.6(0.31)$ & 0.18 & 0.15 & 0.32 \\
\hline Thickness of a fibre, $\mathrm{nm}$ & $1892(12.1)$ & $1916(20.5)$ & 0.42 & 0.64 & 0.40 \\
\hline Curl of a fibre ${ }^{b}$ & $909.7(3.48)$ & $913.5(5.64)$ & 0.53 & 0.15 & 0.16 \\
\hline \multicolumn{6}{|l|}{ Elastic fibres, layers $2-5^{\mathrm{c}}$} \\
\hline Area covered by fibres ${ }^{\mathrm{b}}$ & $66.3(2.55)$ & $67.7(2.64)$ & 0.95 & 0.65 & 0.86 \\
\hline Number of fibres & $97.1(2.76)$ & $97.1(2.99)$ & 0.89 & 0.32 & 0.52 \\
\hline Area of a single fibre, $\mu \mathrm{m}^{2}$ & $50.7(1.42)$ & $49.2(1.72)$ & 0.42 & 0.41 & 0.35 \\
\hline Length of a fibre, $\mu \mathrm{m}$ & $21.5(0.42)$ & $21.0(0.45)$ & 0.27 & 0.31 & 0.16 \\
\hline Thickness of a fibre, $\mathrm{nm}$ & $2186(13.4)$ & $2145(16.4)$ & 0.15 & 0.12 & 0.21 \\
\hline Curl of a fibre ${ }^{b}$ & $909.1(3.00)$ & $897.9(4.94)$ & 0.15 & 0.14 & 0.15 \\
\hline
\end{tabular}

Values are given as mean (SE). $P$ values are derived from linear regression analysis. Model 1: partner (1) and offspring (0), age and gender, model 2: as model 1 plus external factors (smoking, alcohol and sun damage), model 3: as model 2 plus health status (no. of co-morbidities, no. of medication categories). Estimates of the different models are given in supplementary Table 2

${ }^{\mathrm{a}} N=286$

$\mathrm{b} \times 10^{3}$

${ }^{\mathrm{c}} N=277$

$E D J$ epidermal-dermal junction, $S E B$ stratum corneum epidermis boundary

thickness and flattening with chronological age. In the reticular dermis, the number of elastic fibres was increased with chronological age. None of the other morphometric characteristics were related to chronological age in our study population. Furthermore, none of the morphometric epidermal and elastic fibre characteristics differed in offspring from nonagenarian siblings, genetically enriched for familial longevity and their partners as environmentally matched controls. The Framingham CVD risk scores did associate with some characteristics of the papillary dermis; however, other epidermal and elastic fibre morphometric characteristics did not clearly associate with the Framingham CVD risk scores.

The Leiden Longevity Study was designed as a case-control study. We have previously demonstrated that the middle-aged offspring from long-lived nonagenarian siblings have a healthier metabolic profile, a lower prevalence of cardiovascular disease and a lower mortality rate compared to their partners (Schoenmaker et al. 2006; Westendorp et al. 2009). Thus, this unique study design enables the study of longevity-related factors that are present at middle age.

We first examined whether skin morphometric parameters of the upper inner arm were dependent on the chronological age of the donor. Consistent with the literature, we were able to show a deterioration of the epidermal structure with chronological age consisting of a reduced thickness and a flatter appearance (Fenske and Lober 1986; Lavker et al. 1986; Fenske and Conard 1988; Kurban and Bhawan 1990). In addition, we have further demonstrated that this relationship remains after adjustment for potential confounders. For elastin morphology, a distinction was made between papillary and reticular dermis, as 
fibroblasts from upper (papillary), mid and lower (reticular) dermal fibroblasts were observed to differentially express elastin in vitro (Tajima and Izumi 1996). An increase in the number, curl, area and length of the fibres were found in the papillary dermis, the latter two findings supporting a previous report on elastin morphology in the upper inner arm (Robert et al. 1988). These associations disappeared after adjustment for potential confounders. In the reticular dermis (layers 2 to 5), a significant increase in the area covered by the elastic fibres with chronological age was found, supporting previous findings (Kurban and Bhawan 1990; Gogly et al. 1997; Robert et al. 1988; Frances et al. 1990). While we found that the number of fibres significantly increased with chronological age, even after adjustment for potential confounders, we did not find a significant difference in fibre length, contradicting previous reports (Frances et al. 1990; Robert et al. 1988). These discrepancies might be due to different methodologies used; another cause might be the limited age range of our subjects.

A higher perceived age has been shown to be partly genetically determined and to be related with mortality (Christensen et al. 2009; Gunn et al. 2009). In addition, skin wrinkling at the upper inner arm has been linked to health status (Purba et al. 2001). Therefore, we next examined whether the histological changes of the skin serve as a marker of biological age, as defined by familial longevity and Framingham CVD risk. The Framingham CVD risk score is one the most established measures of disease outcome (D'Agostino et al. 2008). In addition, elastin accumulates in atherosclerotic carotid plaques (Goncalves et al. 2003), and it can bind to LDL particles in the arterial wall (Noma et al. 1981). Furthermore, increased arterial stiffening has been linked to properties of elastin (Astrand et al. 2011), and elastin morphology at the upper inner arm has been shown to correlate with that in temporal arteries (Gogly et al. 1998). Thus, we examined whether epidermal or elastic fibre morphometric characteristics were related to CVD risk. The Framingham CVD risk scores associated with the average area and length of elastin fibres in the papillary dermis, even after adjustment for potential confounders. This is the first time that elastin morphology in the skin has been linked to CVD disease risk. However, the decrease in area and length of elastin fibres with increased CVD risk was opposite to their tendency to increase with chronological age. Thus, although significant associations between elastin morphology in the skin and CVD risk has been found, replication of these results is warranted.

Previously we have described that skin fibroblasts derived from the offspring of nonagenarian siblings resembled the responses to cellular stress of fibroblast strains from young subjects, i.e. were biologically younger compared to their partners representing the general population (Dekker et al. 2009). Based on these findings, we expected that the biological differences would have been visible in morphometric skin characteristics comparing the skin of offspring from nonagenarian siblings to their partners. However, none of the morphometric epidermal and elastic fibre characteristics differed dependent on familial longevity status.

To the best of our knowledge, our study is the first to address skin morphometric characteristics in relation to familial longevity including 286 middleaged subjects. The large number of very well characterized subjects is a major strength of this study. However, the chronological age range was rather limited, ranging from 44 to 81 years, with most subjects in their 50th or 60th decade of life. This possibly explains the relatively small effects of chronological age on morphometric skin characteristics and could explain why the more subtle effects of familiar longevity were not found. Another strength of the study is the method used to analyse the slides quantitatively to reduce observer variation. However, it cannot be excluded, even after optimal adjustment of the software settings that measurements such as the morphology of elastic fibres were determined without some technical variability. It is therefore possible that the subtle effects dependent on biological age could not be detected. To minimize any technical influences all measurements were performed in batches including equal numbers of offspring and partner as well as young and old subjects. The sampling of biopsies was performed under highly standardized conditions. However, we cannot fully exclude that skin tissue may have been slightly altered due to the excision procedure. Indeed, during the quality checking procedure, it was noted that some biopsies contained tears, which was suggestive of thin weak skin typically associated with elderly individuals (Branchet et al. 1990). Ethnic differences in skin morphometric characteristics have mainly been linked to photo 
ageing due to differences in pigmentation (Rawlings 2006). In a morphological study of facial skin comparing black and white women, differences in several histological aspects of the skin such as epidermal focal atrophy and more dermal elastic fibres in skin obtained from white women compared to black women have been described (Montagna and Carlisle 1991). Another study has reported a more curled epidermaldermal junction and a slightly thicker epidermis in black skin but no apparent differences in collagen and elastic fibre architectural organization in the dermis (Girardeau et al. 2009). In order to rule out these possible influences of ethnicity on our results, only Caucasian subjects were included in our study.

All biopsies were taken from the inner site of the upper arm in a highly standardized procedure to ensure maximal comparability of the data. Although seasonal effects on thickness of the epidermis have been previously described (Akasaka et al. 2002), these effects did not apply on the analysis of biological age (familial longevity), as skin biopsies of the offspring-partner couples were taken on the same day.

Unlike many previous reports examining skin epidermal and elastic fibre morphology, a number of potential confounders were taken into consideration in our analysis. Skin biopsies were taken from the upper inner arm to ensure minimal UV damage. Since the skin might still have been exposed to some UV light during its lifetime, we additionally adjusted for sun-damage scores obtained from facial photographs of the subjects. The effects of sun damage are also termed extrinsic ageing and are superimposed on the effects of intrinsic (chronological) ageing. Photo-exposed skin shows a broad band of elastic fibres in the papillary dermis including the upper part of the reticular dermis late in life. In the mid and deep reticular dermis, the effect of sun damage is negligible (El-Domyati et al. 2002). Smoking is also a known possible confounder of skin morphometric characteristics, as the relative area, thickness and number of elastic fibres were observed to be increased in non-sun-exposed skin of smokers compared to non-smokers (Frances et al. 1991; Just et al. 2007). We observed changes in the association of chronological or biological age and skin morphometric characteristics when adjusting for external factors, indicating that external factors are closely linked to these morphometric characteristics. Moreover, several diseases have previously been linked to altered skin integrity such as the systemic disease diabetes mellitus type 2 due to increased cross-linking of proteins such as collagen (Wulf et al. 2004). Facial wrinkling has also been associated with COPD and smoking (Patel et al. 2006) and skin wrinkling at the upper inner arm to health status (Purba et al. 2001). Since the association between morphometric characteristics and chronological age weakened after adjustment for disease status, chronological age might not be the most important driver for deterioration of measured epidermal and elastic fibre characteristics; these may have largely been driven by external factors and health status. In support of this, in healthy middle-aged subjects, an association between cardiovascular disease risk and several histological skin characteristics was found.

In conclusion, typical changes of skin morphometric parameters were seen with chronological age. A slower rate of skin ageing was not found in offspring from nonagenarian siblings compared to their partners. Epidermal and elastic fibre morphometric characteristics, as measured from skin biopsies, are therefore not a potential biomarker for familial longevity in middle-aged subjects. However, a link between elastic fibre morphology in the papillary dermis and CVD risk indicates that some aspects of skin morphometric characteristics are markers of health status. Further studies should concentrate on which particular aspects of health and CVD risk relate to skin morphometric characteristics to identify the mechanisms responsible for these links.

Acknowledgments We thank C. de Koning-Treurniet and J. Blom for their help in the laboratory, T. Griffiths, S. Ogden and C. E.M. Griffiths for generating the photo/sun-damage scores and W. Parish for valuable advice and for quality checking the biopsies.

Open Access This article is distributed under the terms of the Creative Commons Attribution Noncommercial License which permits any noncommercial use, distribution, and reproduction in any medium, provided the original author(s) and source are credited.

\section{References}

Akasaka T, Yoshida A, Fukuda S, Takeuchi T, Katsuzaki N (2002) Yearly changes in the physiological function of the skin. Environ Dermatol 9:1-10

Astrand H, Stalhand J, Karlsson J, Karlsson M, Sonesson B, Lanne $\mathrm{T}$ (2011) In vivo estimation of the contribution of elastin and collagen to the mechanical properties in the human abdominal aorta: effect of age and sex. J Appl Physiol 110:176-187 
Branchet MC, Boisnic S, Frances C, Robert AM (1990) Skin thickness changes in normal aging skin. Gerontology 36:28-35

Christensen K, Thinggaard M, McGue M, Rexbye H, Hjelmborg JV, Aviv A, Gunn D, van der Ouderaa F, Vaupel JW (2009) Perceived age as clinically useful biomarker of ageing: cohort study. BMJ 339:b5262

D’Agostino RB Sr, Vasan RS, Pencina MJ, Wolf PA, Cobain M, Massaro JM, Kannel WB (2008) General cardiovascular risk profile for use in primary care: the Framingham Heart Study. Circulation 117:743-753

Dekker P, Maier AB, van Heemst D, de Koning-Treurniet C, Blom J, Dirks RW, Tanke HJ, Westendorp RG (2009) Stress-induced responses of human skin fibroblasts in vitro reflect human longevity. Aging Cell 8:595-603

El-Domyati M, Attia S, Saleh F, Brown D, Birk DE, Gasparro F, Ahmad H, Uitto J (2002) Intrinsic aging vs. photoaging: a comparative histopathological, immunohistochemical, and ultrastructural study of skin. Exp Dermatol 11:398-405

Fenske NA, Conard CB (1988) Aging skin. Am Fam Physician 37:219-230

Fenske NA, Lober CW (1986) Structural and functional changes of normal aging skin. J Am Acad Dermatol 15:571-585

Frances C, Branchet MC, Boisnic S, Lesty CL, Robert L (1990) Elastic fibers in normal human skin. Variations with age: a morphometric analysis. Arch Gerontol Geriatr 10:57-67

Frances C, Boisnic S, Hartmann DJ, Dautzenberg B, Branchet MC, Charpentier YL, Robert L (1991) Changes in the elastic tissue of the non-sun-exposed skin of cigarette smokers. Br J Dermatol 125:43-47

Girardeau S, Mine S, Pageon H, Asselineau D (2009) The Caucasian and African skin types differ morphologically and functionally in their dermal component. Exp Dermatol 18:704-711

Gogly B, Godeau G, Gilbert S, Legrand JM, Kut C, Pellat B, Goldberg M (1997) Morphometric analysis of collagen and elastic fibers in normal skin and gingiva in relation to age. Clin Oral Investig 1:147-152

Gogly B, Godeau G, Septier D, Hornebeck W, Pellat B, Jeandel C (1998) Measurement of the amounts of elastic fibers in the skin and temporal arteries of healthy aged individuals by automated image analysis. Gerontology 44:318-323

Goncalves I, Moses J, Dias N, Pedro LM, Fernandes e Fernandes J, Nilsson J, Ares MP (2003) Changes related to age and cerebrovascular symptoms in the extracellular matrix of human carotid plaques. Stroke 34:616-622

Griffiths CE, Wang TS, Hamilton TA, Voorhees JJ, Ellis CN (1992) A photonumeric scale for the assessment of cutaneous photodamage. Arch Dermatol 128:347-351

Gunn DA, Rexbye H, Griffiths CE, Murray PG, Fereday A, Catt SD, Tomlin CC, Strongitharm BH, Perrett DI, Catt M, Mayes AE, Messenger AG, Green MR, van der Ouderaa F, Vaupel JW, Christensen K (2009) Why some women look young for their age. PLoS One 4:e8021
Just M, Ribera M, Monso E, Lorenzo JC, Ferrandiz C (2007) Effect of smoking on skin elastic fibres: morphometric and immunohistochemical analysis. Br J Dermatol 156:85-91

Kurban RS, Bhawan J (1990) Histologic changes in skin associated with aging. J Dermatol Surg Oncol 16:908-914

Lavker RM, Zheng PS, Dong G (1986) Morphology of aged skin. Dermatol Clin 4:379-389

Montagna W, Carlisle K (1991) The architecture of black and white facial skin. J Am Acad Dermatol 24:929-937

Noma A, Takahashi T, Wada T (1981) Elastin-lipid interaction in the arterial wall. Part 2. In vitro binding of lipoproteinlipids to arterial elastin and the inhibitory effect of high density lipoproteins on the process. Atherosclerosis 38:373-382

Patel BD, Loo WJ, Tasker AD, Screaton NJ, Burrows NP, Silverman EK, Lomas DA (2006) Smoking related COPD and facial wrinkling: is there a common susceptibility? Thorax 61:568-571

Purba MB, Kouris-Blazos A, Wattanapenpaiboon N, Lukito W, Rothenberg E, Steen B, Wahlqvist ML (2001) Can skin wrinkling in a site that has received limited sun exposure be used as a marker of health status and biological age? Age Ageing 30:227-234

Rawlings AV (2006) Ethnic skin types: are there differences in skin structure and function? Int J Cosmet Sci 28:79-93

Robert C, Lesty C, Robert AM (1988) Ageing of the skin: study of elastic fiber network modifications by computerized image analysis. Gerontology 34:291-296

Schoenmaker M, de Craen AJ, de Meijer PH, Beekman M, Blauw GJ, Slagboom PE, Westendorp RG (2006) Evidence of genetic enrichment for exceptional survival using a family approach: the Leiden Longevity Study. Eur J Hum Genet 14:79-84

Tajima S, Izumi T (1996) Differential in vitro responses of elastin expression to basic fibroblast growth factor and transforming growth factor beta 1 in upper, middle and lower dermal fibroblasts. Arch Dermatol Res 288:753-756

Warren R, Gartstein V, Kligman AM, Montagna W, Allendorf RA, Ridder GM (1991) Age, sunlight, and facial skin: a histologic and quantitative study. J Am Acad Dermatol 25:751-760

Westendorp RG, van Heemst D, Rozing MP, Frolich M, Mooijaart SP, Blauw GJ, Beekman M, Heijmans BT, de Craen AJ, Slagboom PE (2009) Nonagenarian siblings and their offspring display lower risk of mortality and morbidity than sporadic nonagenarians: the Leiden Longevity Study. J Am Geriatr Soc 57:1634-1637

Wijsman CA, Rozing MP, Streefland TC, Le CS, Mooijaart SP, Slagboom PE, Westendorp RG, Pijl H, van Heemst D (2010) Familial longevity is marked by enhanced insulin sensitivity. Aging Cell 10(1):114-121

Wulf HC, Sandby-Moller J, Kobayasi T, Gniadecki R (2004) Skin aging and natural photoprotection. Micron 35:185191 